\title{
Review on DIS electroproduction on nuclei
}

\section{N. Bianchi ${ }^{\mathrm{a}}$}

${ }^{a}$ INFN Laboratori Nazionali di Frascati

Via E. Fermi 40, I - 00044 Frascati, Italy

The inclusive Deep-Inelastic-Scattering (DIS) of high energy leptons on free nucleons has been used from late 60 s to measure the partonic distributions and, by using nuclear targets, to study the medium modification to these distributions. Semi-Inclusive DIS (SIDIS) of high energy leptons on free nucleons can be used to study another fundamental property of the quarks, the fragmentation function. This function can be also modified by the nuclear medium and can be revealed by performing SIDIS measurements on nuclear targets. These measurements have been recently performed by HERMES with a 27.5 $\mathrm{GeV}$ positron beam and at lower energy by CLAS. The possible interpretations in terms of medium modifications of the parton fragmentation function and the implications on the parton energy loss are discussed. Similar hadron quenching appeared from Au-Au central collisions at RHIC. Despite the two different regimes, both these observations have been interpreted in terms of in medium parton energy loss which depends on the density of the medium. A challenge in this research field, will be represented by the measurement of the jet quenching in a hot and dense medium like the Quark Gluon Plasma which is expected to be created at the LHC energy. For this purpose, an electromagnetic calorimeter to complement the excellent performance of the ALICE detector has been proposed.

\section{Fragmentation functions and multiplicities}

The SIDIS process can be factorized in a parton distribution function $q_{f}$, which is function of the Bjorken scaling variable $x=Q^{2} /(2 M \nu)$, a hard scattering cross section $d \sigma_{f}$ calculable in QCD, and a parton fragmentation function $D_{f}^{h}$. The fragmentation function $D_{f}^{h}(z)$ represents the probability for a quark of flavor $f$ to fragment into a specific hadron $h$ carrying a fraction $z=E_{h} / \nu$ of the energy of the struck quark. In analogy with the parton distribution functions, the fragmentation functions depend on a scaling variable $z$ and their logarithmic $Q^{2}$-evolution is determined by the DGLAP equations.

In the Quark Parton Model (QPM) description of SIDIS the fragmentation functions are multiplied by the parton distributions, and experimental hadron multiplicities $M^{h}$ are determined by normalizing the SIDIS yield to the DIS rate:

$$
M^{h}=\frac{1}{N_{D I S}} \frac{d N^{h}(x, z)}{d z}=\frac{\sum_{f} e_{f}^{2} q_{f}(x) D_{f}^{h}(z)}{\sum_{f} e_{f}^{2} q_{f}(x)}
$$

It is easy to demonstrate that within the QPM and under the $u$-quark dominance assumption, the pion multiplicities are almost equivalent to the pion fragmentation func- 


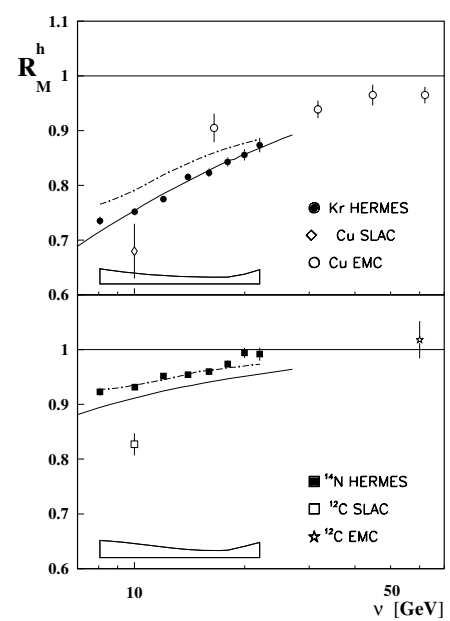

Figure 1. Multiplicity ratios as a function of $\nu$ for hadrons with $z>0.2$ for HERMES, SLAC and EMC. The solid curves are calculations from [5] and the dot-dashed curves are calculations from $[6]$

tions. In QCD the relation between fragmentation functions and multiplicities is more complicated since both the leading order (LO) and the next to leading order (NLO) terms of the cross section are convoluted with parton distributions and with the hadron fragmentation functions. Nevertheless, it has been shown [1] that, once integrating over a broad range of $x$, the SIDIS multiplicities are in good agreement with the NLO-QCD evolution of fragmentation functions determined from $e^{+} e^{-}$measurements at LEP. This result suggests the validity of the factorization assumption for the SIDIS process and of the approximation of fragmentation functions with multiplicities.

\section{Hadronization in the nuclear medium}

The study of the medium modification of fragmentation functions can be performed by SIDIS measurements on nuclear targets where the nucleus acts as an ensemble of targets which reduce the multiplicity of fast hadrons due to both partonic and hadronic interactions. The recent results from HERMES $[2, ?, ?]$ provided a new strong boost of interest in this field. HERMES is an experiment at DESY which is mainly devoted to the study of the spin structure of the nucleon by using a polarized positron (or electron) beam and polarized internal gas targets. In addition, some HERMES runs have been performed with unpolarized nuclear gas targets ( $\left.\mathrm{D},{ }^{4} \mathrm{He}, \mathrm{N}, \mathrm{Ne}, \mathrm{Kr}, \mathrm{Xe}\right)$ with densities up to $\sim 10^{16}$ nucl $\cdot \mathrm{cm}^{-2}$, allowing the extension of the physics program to the study of several nuclear effects. In fig. 2 the HERMES results for the fast hadron multiplicity 


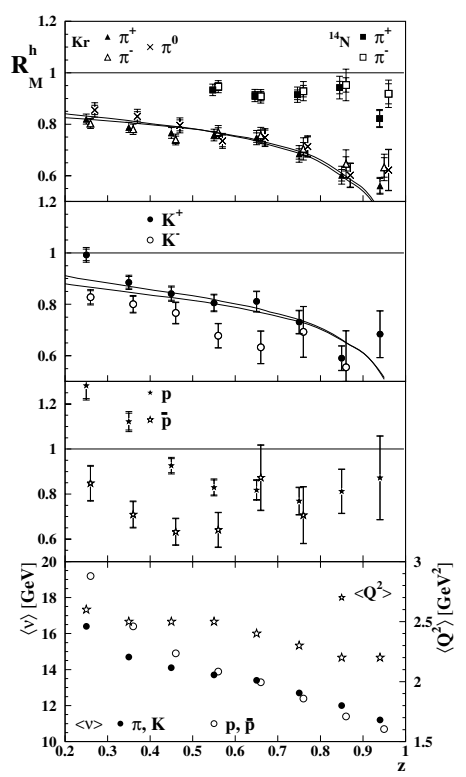

Figure 2. HERMES multiplicity ratio $\mathrm{Kr} / \mathrm{D}$ for different charged hadrons as a function of the scaling variable $z$. In the lower panel the relevant average kinematic variables are presented for $z$-bin. The thick (thin) solid curves represent the calculations from [5] for positive (negative) charge states.

ratios from $\mathrm{N}$ and $\mathrm{Kr}$ relative to that from deuterium, are shown in comparison with data from earlier experiments. As it is seen, the HERMES kinematic is well suited to study quark propagation and hadronisation, and the results indicate an increase of the multiplicity ratio (thus a decrease of the medium effect) with $\nu$, in agreement with the higher energy EMC data.

The complete particle identification in HERMES allows to fully disentangle the information for different hadron types. This is shown in Fig. 2 where the multiplicity ratios between Kr and D nuclei are presented as function of the scaling variable $z$. As it is seen, the medium effects for $\pi^{+}$and $\pi^{-}$are equal. Also the medium effects on $K^{-}$production are similar to the charged pion case. Quite interesting is the difference between $\mathrm{K}^{+}$and $K^{-}$and especially between $p$ and $\bar{p}$. In particular the effect for the proton is strongly different at low- $z$, where a contamination from the target fragmentation can contribute. The different results for different hadrons may reveal differences in the modification of $q$ and $\bar{q}$ fragmentation functions, in the hadron formation times and in the different hadronic interaction cross sections. Fig. 3 shows new preliminary HERMES results obtained on different nuclei. These data suggest a dependence of the nuclear attenuation $\left(1-R_{A}^{\pi}\right) \approx A^{2 / 3}$, i.e. proportional to the square of the nuclear size. A decreasing nuclear 

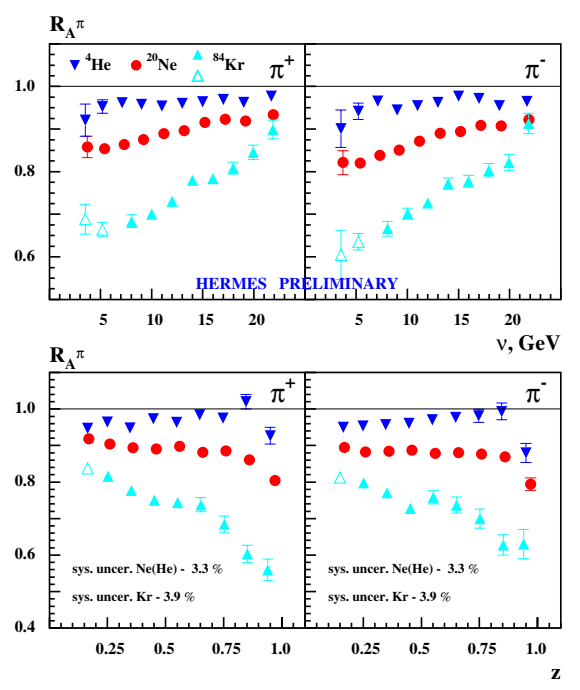

Figure 3. HERMES preliminary multiplicity ratio for $\pi^{+}$and $\pi^{-}$from ${ }^{4} \mathrm{He},{ }^{20} \mathrm{Ne}$ and ${ }^{84} \mathrm{Kr}$ targets. The open triangles for the Kr target correspond to extended kinematics with respect to published $\mathrm{Kr}$ data.

effect with the increasing $Q^{2}$ can be also derived from these preliminary data. Both these two experimental findings indicate a modification of the fragmentation function.

\section{Modification of fragmentation functions}

The new HERMES results have been interpreted with theoretical models describing hadron production on nuclei in terms of a modification of the quark fragmentation functions.

In Ref. [6] the modification of quark fragmentation functions and their QCD evolution are described in the framework of multiple parton scattering. As consequence of the partonic energy loss $d E / d x$ due to the induced gluon radiation, the fragmentation function in the nucleus $D^{A}\left(z, Q^{2}\right)$ is modified compared to the one in deuterium $D^{D}\left(z, Q^{2}\right)$. In the framework of this calculation, for a cold and static system like the nucleus, the quark energy loss involved in SIDIS reaction has been determined. The extracted energy loss is $d E / d x \approx 0.5 \mathrm{GeV} / \mathrm{fm}$ for a quark with $\mathrm{E}=10 \mathrm{GeV}$ in an $\mathrm{Au}$ nucleus. Including the effect of the expansion, an increase of the gluon density of a factor $\sim 30$ has been derived for the initial hot stage of $A u+A u$ collisions at RHIC at $\sqrt{s}=130 \mathrm{GeV}$. A theoretical description of the HERMES data in terms of in-medium distribution of the parton energy loss has been also recently given in Ref. [7]. The calculation quite well reproduce the HERMES data, and the derived energy loss per unit length $d E / d x=0.62 \mathrm{GeV} / \mathrm{fm}$ is close to the result of Ref. [6].

In Ref. [8] the combined effect of the fragmentation function modification due to the 
induced gluon radiation and of the hadron interaction inside the nucleus has been calculated. The contribution due to the interaction of the pre-formed hadron in the nuclear medium was claimed to be large.

Also in Ref. [5] the nuclear absorption of the pre-formed hadrons is seen to significantly affect the hadron production on heavy nuclei in the kinematic region of the HERMES experiment. In this work the modification of quark fragmentation functions have been considered in terms of $Q^{2}$-rescaling model, originally developed to interpret the EMCeffect in the nuclear structure functions, and of absorption of the produced hadrons. The deconfinement scale is assumed to be proportional to the degree of overlap of the nucleons inside the given nucleus.

In Ref. [9] the data analysis was carried out in the framework of a probabilistic coupledchannel transport model which allowed for a treatment of the final-state interactions beyond simple absorption mechanisms. Furthermore, the event by event simulations accounted for the kinematics cuts of the experiment as well as the geometrical acceptance of the detectors. This study suggested that the pre-hadronic final state interactions play a dominant role in the kinematic regime of HERMES while the approach overestimate the small attenuation observed at EMC energies.

\section{Modification of $p_{t}$ distribution}

The scattering of quark and gluon increases the transverse momentum of the struck quark compared to its intrinsic value and, as consequence, the transverse momentum $p_{t}$ of the leading hadron. This effect is known as the Cronin effect [10] and has previously been observed in many hadronic reactions like in $p A$ collisions or in high energy heavy-ion collisions performed at CERN SPS. A nuclear enhancement at high $p_{t}^{2}$ is also observed in the HERMES data as shown in Fig.4. The HERMES data for $p_{t}^{2}<1 \mathrm{GeV}^{2}$ indicate the hadron attenuation discussed above, while the data for $p_{t}^{2} \geq 1 \mathrm{GeV}^{2}$ reflect the $p_{t}$-broadening that can be considered as clean evidence of parton and hadron multiple scattering in the final state. The increase of $p_{t}$ is larger for the heavier nucleus. The $p_{t}$ broadening in SIDIS is predicted [11] to dependent on the multi-parton correlation functions inside a nucleus and to be proportional to the nuclear radius.

\section{Expectations from Jlab}

Given the relatively low energy of Jlab (presently $6 \mathrm{GeV}$ at maximum) the factorization of SIDIS into $x$-dependent distribution functions and $z$-dependent fragmentation functions maybe questionable. Promising test of this assumption has been performed comparing the yield in the electroproduction of $\pi^{-}$with a Monte-Carlo based on LO $x-z$ factorization. The CLAS collaboration has already collected data for hadron production for different nuclei. The present data under analysis are in the kinematic region up to $Q^{2} \sim 4 \mathrm{GeV}^{2}$ and $\nu \sim 5 \mathrm{GeV}$. With the planned $12 \mathrm{GeV}$ Jlab upgrade the above ranges will be enlarge up to $Q^{2} \sim 9 \mathrm{GeV}^{2}$ and $\nu \sim 9 \mathrm{GeV}$. An other experiment has been approved in Hall-A (E04-002) with the purpose of a high precision measurement at large $z$ for fixed kinematics. 


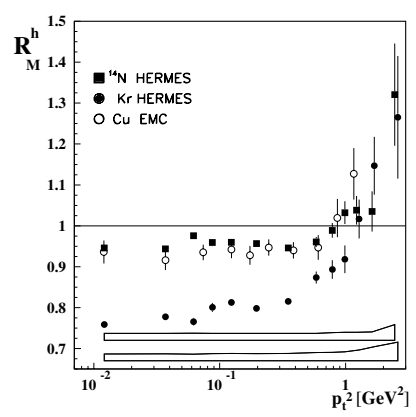

Figure 4. $p_{t}$-dependence of the nuclear multiplicity ratio in SIDIS.

\section{Hadron suppression RHIC}

Two of the most outstanding results from RICH are the leading hadron suppression at large $p_{t}$ (see [12] for a summary) and the washing out of the jet-like azimuthal correlations [13]. Fig. 5 shows the suppression of hadrons in central collisions. The data are compared with PQCD predictions where the created medium is parametrized by a gluon transport coefficient $q=\mu^{2} / \lambda$ where $\mu$ is the typical momentum transfer and $\lambda$ is the gluon mean free path. Therefore a large hadron suppression indicates a large medium density formed in the early stage after the collision. It is to point out that, in the same $p_{t}$ range, the photons are not suppressed like the hadrons. Unfortunately, the $p_{t}$ range explored by RHIC is not sufficient to study PQCD evolution. In addition, the measurement of the leading hadron only can provide only approximative lower limit on the parton energy loss. The study of the azimuthal correlations of jet-like events also shows that the hadrons produced in the opposite direction of the detected leading high- $p_{t}$ hadron are strongly suppressed by the dense medium while hadrons produced in the same jet-cone of the leading hadrons are not. However the picture emerging from the experimental results at RHIC is that the state produced in nucleus-nucleus collision at these energies shows a behavior hardly compatible with the one expected for an ideal gas of free quarks and gluons. It rather looks like a liquid, with a short mean free-path and a large opacity as shown by the suppression of particle production at high $p_{t}$.

\section{Jet quenching at ALICE}

RHIC results on leading hadron suppression indicate that most of the energy lost by the leading parton remains within the jet cone, but several questions on the medium modification of the jet structure have not been addressed. These include the longitudinal and transverse structures of the quenched jet, the associated radiation observables, the dependence on the parton flavor. These topics will be studied by ALICE thanks to both the robustness of its tracking (excellent performance for charged particles both at low and high $p_{t}$ from the Time Projection Chamber) and the charged particle identification 


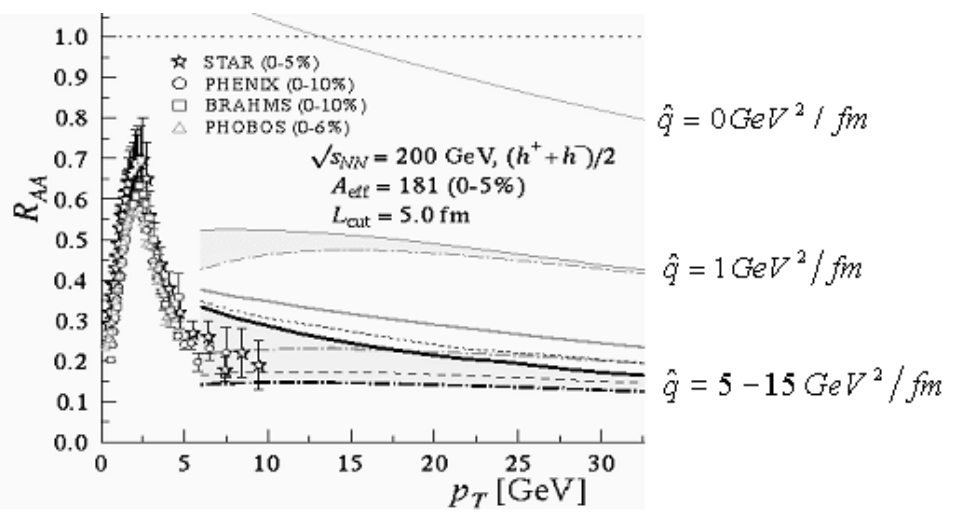

Figure 5. Hadron suppression in Au-Au collision at RHIC. Curves are pQCD prediction based on parton energy loss with different assumptions on the gluon transport coefficient in the medium.

systems. Large effects are expected in both the low $p_{t}$ and in the high $p_{t}$ regions. To make ALICE better suited for jet physics, the performances on high $p_{t}$ particles and jets can be significantly improved by completing the present set-up with a large Electromagnetic Calorimeter (EmCal) [14]. This would significantly improve the resolution on the jet energy and on the particle composition (with the detection of both charged and neutral particles). It would also allow to "calibrate" the jet energy by measuring the high energy photon emitted in the opposite direction ( $\gamma$-jet physics). EmCal would be used to trigger on the jet energy itself, thus allowing a significant improvement of the statistics achievable for jets of high energy. Finally, due too both the $\gamma / \pi^{0}$ and the electron/hadron discrimination, EmCal will enhance the ALICE capabilities at high $p_{t}$ for direct photon and heavy quarks $\left(c, b \rightarrow e^{+} e^{-}\right)$measurements. Fig. 6 shows the annual yields expected in one year of $\mathrm{Pb}-\mathrm{Pb}$ minimum bias events. Assuming a required statistics of $10^{4}$ events to be collected, the inclusive jet can be studied up to $p_{t}$ of about $200 \mathrm{GeV}$. Dijets (one in EmCal and the other partially reconstructed only in the TPC) up to $170 \mathrm{GeV}$. Single $\pi^{0}$ and $\gamma$ up to about 75 and $45 \mathrm{GeV}$, respectively. Positrons and electrons from charm and bottom decay up to $25 \mathrm{GeV}$.

The proposed EmCal will cover the rapidity range of $-0.7<\eta<+0.7$ and the azimuthal angle $\Delta \phi=110^{\circ}$. It will be positioned back to back to the smaller PHOS calorimeter. The calorimeter will be compose by more than $1.210^{4}$ channels since a high granularity and a large angular coverage are required for the jet reconstruction. The technology will be the shashlik sampling calorimetry with alternate thin layers of lead and scintillator tiles. Each counter will be tapered of about $1.5^{\circ}$ in order to achieve a projective configuration in the rapidity variable. The optical readout will be performed with Wave Length Shifter fibers coupled with Avalanche Photo Diodes. The preamplifiers and the front end electronics will be similar to the ones already planned for the PHOS de- 


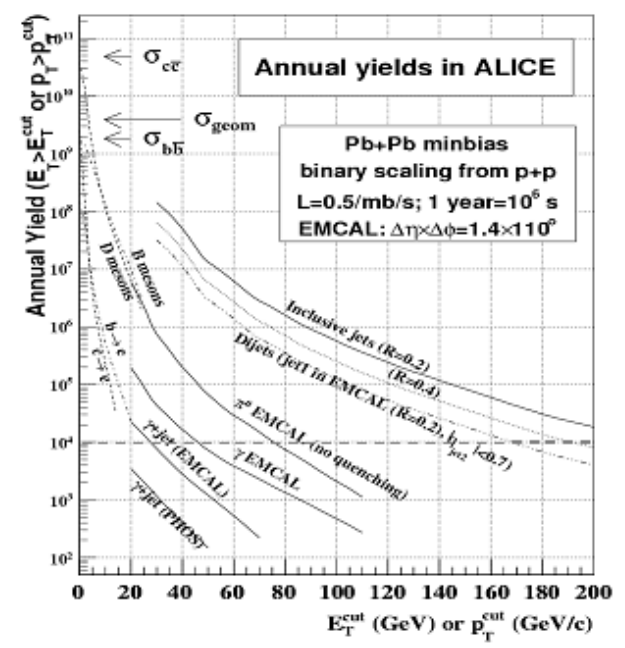

Figure 6. Rates for different hard processes expected in ALICE for a $\mathrm{Pb}-\mathrm{Pb}$ data taking time of $10^{6}$ (this value corresponds to the $\mathrm{Pb}-\mathrm{Pb}$ running time per year) with the proposed EmCal. Horizontal line shows the statistic lower limit of $10^{4}$ events per year

tector. The expected performances are an energy resolution better than $10 \% / \sqrt{E(G e V)}$, a spatial resolution better than $1 \mathrm{~cm}$, a $\gamma / \pi^{0}$ separation up to about $50 \mathrm{GeV}$.

EmCal is proposed to ALICE by a about 18 groups from France, Italy, Russia and USA with the aim to be completed and commissioned in 2010.

\section{REFERENCES}

1. A. Airapetian et al., Eur. Phys. J. C 21 (2001) 599

2. A. Airapetian et al., Eur. Phys. J. C 20 (2001) 479

3. A. Airapetian et al., Phys. Lett. B 577 (2003) 37

4. A. Airapetian et al., Phys. Rev. Lett. 96 (2006) 162301

5. A. Accardi, V. Muccifora and H.J. Pirner, Nucl. Phys. A 720 (2003) 131

6. E. Wang and X.N. Wang, Phys. Rev. Lett. 89 (2002) 162301

7. F. Arleo, Eur. Phys. J. C 30 (2003) 213

8. B. Kopeliovich et al., Nucl. Phys. A 740 (2004) 211

9. T. Falter et al., Phys. Rev. C 70 (2004) 054609

10. J.W. Cronin, Phys. Rev. D 11 (1975) 3105

11. X. Guo and J. Qiu, Phys. Rev. D 61 (2000) 096003

12. J. L. Klay, J. Phys. G 31 (2005) S451

13. J. Adams et al., Phys. Rev. Lett. 91 (2003) 072304

14. CERN-LHCC-2006-014 